\title{
ON SUPPORT POINTS OF THE CLASS $S^{0}\left(B^{n}\right)$
}

\author{
SEBASTIAN SCHLEISSINGER
}

(Communicated by Franc Forstnerič)

\begin{abstract}
We consider support points of the class $S^{0}\left(B^{n}\right)$ introduced by G. Kohr and prove that, given a normalized Loewner chain $f(z, t)$ such that $f(\cdot, 0)$ is a support point of $S^{0}\left(B^{n}\right)$, all elements of the chain are support points of $S^{0}\left(B^{n}\right)$. Also, we prove a similar result for Loewner chains that come from the Roper-Suffridge extension operator.
\end{abstract}

\section{INTRODUCTION AND RESUlts}

Let $D \subset \mathbb{C}^{n}$ be a domain. We denote by $\mathcal{H}\left(D, \mathbb{C}^{n}\right)$ the set of all holomorphic functions which map from $D$ to $\mathbb{C}^{n}$. Furthermore we denote by $\|z\|$ the Euclidean norm of $z \in \mathbb{C}^{n}$. Let $B^{n}=\left\{z \in \mathbb{C}^{n} \mid\|z\|<1\right\}$ be the unit ball and let $S\left(B^{n}\right)$ be the set of all normalized biholomorphic mappings on $B^{n}$, i.e.,

$$
S\left(B^{n}\right):=\left\{f \in \mathcal{H}\left(B^{n}, \mathbb{C}^{n}\right) \mid f \text { biholomorphic with } f(0)=0, D f(0)=I_{n}\right\} .
$$

Compared to the case $n \geq 2$, the class $S:=S\left(B^{1}\right)$ is well understood, and a powerful tool to describe this class is the so-called Loewner theory: every element $f$ of $S$ can be embedded in a Loewner chain, which can be described by a differential equation. This equation, in turn, can be used to study properties of $f$. In higher dimensions, the situation is more complicated and G. Kohr introduced the subclass $S^{0}\left(B^{n}\right) \subseteq S\left(B^{n}\right)$ in Koh01 by the method of Loewner chains in order to get a class having similar properties as $S$.

We recall the definition and some properties of $S^{0}\left(B^{n}\right)$ (see [GK03] or GHKK12]). For $f, g \in \mathcal{H}\left(B^{n}, \mathbb{C}^{n}\right)$, we write $f \prec g$ if there exists a Schwarz mapping $v \in \mathcal{H}\left(B^{n}, \mathbb{C}^{n}\right)$ (i.e., $\|v(z)\| \leq\|z\|$ for all $z \in B^{n}$ ) such that $f=g \circ v$.

A mapping $f: B^{n} \times[0, \infty) \rightarrow \mathbb{C}^{n}$ is called a normalized Loewner chain if $e^{-t} f(\cdot, t) \in S\left(B^{n}\right)$ for all $t \geq 0$ and $f(\cdot, s) \prec f(\cdot, t)$ for all $s, t$ with $0 \leq s \leq t$.

A mapping $f \in S\left(B^{n}\right)$ is said to have parametric representation if there exists a normalized Loewner chain $f(z, t)$ with $f=f(\cdot, 0)$ such that $\left\{e^{-t} f(\cdot, t)\right\}_{t \geq 0}$ is a normal family on $B^{n} . S^{0}\left(B^{n}\right)$ is the set of all biholomorphic mappings on $B^{n}$ which have parametric representation.

When $n=1$, the normalization in those Loewner chains is not really restrictive and we have $S^{0}\left(B^{1}\right)=S$. For $n \geq 2$, however, we always have $S^{0}\left(B^{n}\right) \subsetneq S\left(B^{n}\right)$. On the other hand, $S^{0}\left(B^{n}\right)$ has some nice properties similar to $S$; e.g., it is compact in contrast to $S\left(B^{n}\right)$ and the elements satisfy a growth condition similar to the growth theorem for $S$ (see Corollary 8.3.9 in GK03]). Besides, the class $S^{*}\left(B^{n}\right) \subset S\left(B^{n}\right)$ of normalized starlike mappings is contained in $S^{0}\left(B^{n}\right)$ because $f \in S^{*}\left(B^{n}\right)$ if and

Received by the editors August 21, 2012 and, in revised form, November 26, 2012 and December 3, 2012 .

2010 Mathematics Subject Classification. Primary 32H02.

(C)2014 American Mathematical Society Reverts to public domain 28 years from publication 
only if $e^{t} f(z)$ is a normalized Loewner chain. We denote by $\operatorname{supp} S^{0}\left(B^{n}\right)$ the set of all support points of $S^{0}\left(B^{n}\right)$, i.e., those functions that maximize over $S^{0}\left(B^{n}\right)$ the real part of a continuous linear functional $L: \mathcal{H}\left(B^{n}, \mathbb{C}^{n}\right) \rightarrow \mathbb{C}$, which is nonconstant on $S^{0}\left(B^{n}\right)$. We will prove the following theorem, which is already known to be true for the case $n=1$, i.e., for the class $S$.

Theorem 1.1. Let $f \in \operatorname{supp} S^{0}\left(B^{n}\right)$ and let $f(z, t)$ be a normalized Loewner chain with $f(\cdot, 0)=f$ such that $\left\{e^{-t} f(\cdot, t)\right\}_{t \geq 0}$ is a normal family on $B^{n}$. Then $e^{-t} f(\cdot, t) \in \operatorname{supp} S^{0}\left(B^{n}\right)$ for all $t \geq 0$.

In order to get (nontrivial) examples for biholomorphic mappings for $n \geq 2$, one can use extension operators, which are mappings of the form $\Theta: S\left(B^{k}\right) \rightarrow$ $S\left(B^{k+m}\right), k, m \in \mathbb{N}$. Such an operator is said to preserve normalized Loewner chains if for any normalized Loewner chain $f(z, t)$ on $B^{k}$ the mapping $e^{t} \Theta\left(e^{-t} f(z, t)\right)$ is a normalized Loewner chain on $B^{k+m}$. One example of such an operator is the classical Roper-Suffridge extension operator $\Psi_{n}: S \rightarrow S\left(B^{n}\right)$ with $n \geq 2$ and

$$
\Psi_{n}(f)\left(z_{1}, \ldots, z_{n}\right)=\left(f\left(z_{1}\right), z_{2} \sqrt{f^{\prime}\left(z_{1}\right)}, \ldots, z_{n} \sqrt{f^{\prime}\left(z_{1}\right)}\right)
$$

(see Section 11.3 in GK03). Here, the branch of the square root is chosen such that $\sqrt{1}=1$. We have $\Psi_{n}(S) \subseteq S^{0}\left(B^{n}\right) . \Psi_{n}$ has other nice properties; e.g., it maps starlike/convex/Bloch mappings again onto starlike/convex/Bloch mappings (see Section 11.1 in GK03]).

We will prove Conjecture 3.1 in GKP07, which says that all support points of $\Psi_{n}(S)$ are unbounded mappings. This implies the following result.

Theorem 1.2. Let $f \in S$ and $F=\Psi_{n}(f)$. Also let $f\left(z_{1}, t\right)$ be a normalized Loewner chain with $f(\cdot, 0)=f$ and let $F(z, t)=e^{t} \Psi_{n}\left(e^{-t} f(z, t)\right)$. If $F \in \operatorname{supp} \Psi_{n}(S)$, then $e^{-t} F(\cdot, t) \in \operatorname{supp} \Psi_{n}(S)$ for all $t \geq 0$.

\section{Proof of Theorem 1.1}

We will prove Theorem 1.1 by generalizing ideas from a proof for the case $n=1$, which is described in HM84. First, we need to take a look at Runge domains. Let $D \subseteq \mathbb{C}^{n}$ be a domain of holomorphy. We let $\mathcal{P}\left(D, \mathbb{C}^{n}\right) \subset \mathcal{H}\left(D, \mathbb{C}^{n}\right)$ be the set of all polynomials with the topology induced by locally uniform convergence in $D$.

For $n=1$ (a version of) the polynomial Runge theorem says that $\mathcal{P}(D, \mathbb{C})$ is dense in $\mathcal{H}(D, \mathbb{C})$ whenever $D$ is simply connected. In higher dimensions this is no longer true and one calls $D$ a Runge domain if $\mathcal{P}\left(D, \mathbb{C}^{n}\right)$ is dense in $\mathcal{H}\left(D, \mathbb{C}^{n}\right)$. Furthermore, if $E \subseteq \mathbb{C}^{n}$ is a domain with $D \subseteq E$, then the pair $(D, E)$ is called a Runge pair if $\mathcal{H}\left(E, \mathbb{C}^{n}\right)$ is dense in $\mathcal{H}\left(D, \mathbb{C}^{n}\right)$. So $D$ is a Runge domain if and only if $\left(D, \mathbb{C}^{n}\right)$ is a Runge pair. The unit ball $B^{n}$ is a simple example of a Runge domain. However, the Runge property is not invariant with respect to biholomorphic mappings, as an example of J. Wermer shows; see Wer59.

First, we rephrase the property that $B^{n}$ is mapped biholomorphically onto a Runge domain by $f$ in terms of $f$.

Lemma 2.1. Let $f \in S\left(B^{n}\right)$. The following statements are equivalent:

a) $f\left(B^{n}\right)$ is a Runge domain.

b) $f^{-1} \in \overline{\mathcal{P}\left(f\left(B^{n}\right), \mathbb{C}^{n}\right)}$.

c) For every $g \in \mathcal{H}\left(B^{n}, \mathbb{C}^{n}\right)$ there exists a sequence $p_{k}$ of polynomials with $g(z)=\lim _{k \rightarrow \infty} p_{k}(f(z))$ locally uniformly in $B^{n}$. 
Proof. " $a) \Rightarrow b)$ ": If $f\left(B^{n}\right)$ is Runge, then $f^{-1} \in \mathcal{H}\left(f\left(B^{n}\right), \mathbb{C}^{n}\right)=\overline{\mathcal{P}\left(f\left(B^{n}\right), \mathbb{C}^{n}\right)}$.

"b) $\Rightarrow c)$ ": Let $h_{k}$ be a sequence of polynomials which approximate $f^{-1}$ locally uniformly on $f\left(B^{n}\right)$ and let $g \in \mathcal{H}\left(B^{n}, \mathbb{C}^{n}\right)$. As $B^{n}$ is Runge, there exists a sequence $q_{k}$ of polynomials which approximate $g$ locally uniformly on $B^{n}$. Then $q_{k} \circ h_{k} \circ f$ approximates locally uniformly the map $g \circ f^{-1} \circ f=g$.

"c) $\Rightarrow a)$ ": Note that the linear map $F: \mathcal{H}\left(f\left(B^{n}\right), \mathbb{C}^{n}\right) \rightarrow \mathcal{H}\left(B^{n}, \mathbb{C}^{n}\right), F(g)=$ $g \circ f$, is an isomorphism, and hence locally uniform convergence of a sequence $h_{k}$ in $f\left(B^{n}\right)$ is equivalent to locally uniform convergence of the sequence $h_{k} \circ f$ in $B^{n}$.

Let $f \in S^{0}\left(B^{n}\right)$ and $f(z, t)$ be a normalized Loewner chain with $f=f(\cdot, 0)$ such that $\left\{e^{-t} f(\cdot, t)\right\}_{t \geq 0}$ is a normal family on $B^{n}$. For every $s, t$ with $0 \leq s \leq t$ we have $f(\cdot, s) \prec f(\cdot, t)$ and we can write $f(\cdot, s)=f(v(\cdot, s, t), t)$ with a unique Schwarz mapping $v(\cdot, s, t)$, which is also called transition mapping. For $s=0$ we will just write $v_{t}:=v(\cdot, 0, t)$. For a fixed $s$, the family $\left\{v(\cdot, s, t)_{t \geq s}\right\}$ satisfies the so-called Loewner ordinary differential equation (see GHKK12]).

In $\mathrm{ABFW}$, a remarkable fact concerning solutions to this equation is shown.

Theorem 2.2 (Proposition 5.1 in $[\mathrm{ABFW}]$ ). $v\left(B^{n}, s, t\right)$ is a Runge domain for all $s, t$ with $0 \leq s \leq t$.

We note a simple consequence of the proof of Theorem 2.2 .

Theorem 2.3. Every $f \in S^{0}\left(B^{n}\right)$ maps $B^{n}$ onto a Runge domain.

Proof. Let $f \in S^{0}\left(B^{n}\right)$ and $f(z, t)$ be a normalized Loewner chain with $f=f(\cdot, 0)$ such that $\left\{e^{-t} f(\cdot, t)\right\}_{t \geq 0}$ is a normal family on $B^{n}$. For every $s, t$ with $0 \leq s \leq t$ we have $f(\cdot, s) \prec f(\cdot, t)$, and Theorem 4.2 in ABFW] immediately implies that $\left(f\left(B^{n}, s\right), f\left(B^{n}, t\right)\right)$ is a Runge pair. Let $R$ be the Loewner range of the Loewner chain $f(z, t)$, i.e., $R=\cup_{t \geq 0} f\left(B^{n}, t\right)$. One can easily show that $\left(f\left(B^{n}, t\right), R\right)$ is also a Runge pair for every $t \geq 0$. Now, the Koebe distortion theorem for the class $S^{0}\left(B^{n}\right)$, which is Corollary 8.3.9 in GK03, implies

$$
R=\bigcup_{t \geq 0} f\left(B^{n}, t\right) \supseteq \bigcup_{t \geq 0}\left\{z \in \mathbb{C}^{n} \mid\|z\|<\frac{e^{t}}{4}\right\}=\mathbb{C}^{n},
$$

and consequently $R=\mathbb{C}^{n}$. Hence $\left(f\left(B^{n}, t\right), \mathbb{C}^{n}\right)$ is a Runge pair for every $t \geq 0$. In particular, $f\left(B^{n}\right)=f\left(B^{n}, 0\right)$ is a Runge domain.

Corollary 2.4. For $n \geq 2$, every $f \in S^{0}\left(B^{n}\right)$ can be approximated locally uniformly by automorphisms of $\mathbb{C}^{n}$.

Proof. We just have to apply Theorem 2.1 in [AL92, which states that every biholomorphic map from a starlike domain to a Runge domain can be approximated by automorphisms of $\mathbb{C}^{n}$ when $n \geq 2$.

It would be nice to find out more about those $f \in S^{0}\left(B^{n}\right)$ that extend to automorphisms of $\mathbb{C}^{n}$. When $n=1$, the only normalized univalent function that is also univalent in $\mathbb{C}$ is the identity mapping. Corollary 2.4 suggests that the case $n \geq 2$ should be more interesting.

Remark 2.5. Theorem 2.3 can also be shown without using the Loewner range, namely by proving Corollary 2.4 first: Every $f \in S^{0}\left(B^{n}\right)$ can be calculated by $v_{t}$ via

$$
f(z)=\lim _{t \rightarrow \infty} e^{t} v_{t}(z)
$$


locally uniformly on $B^{n}$; see Theorem 8.1.9 in GK03. By combining Theorem 2.2 with Theorem 2.1 in AL92, we see that $f$ can be approximated locally uniformly by automorphisms of $\mathbb{C}^{n}$. This fact already implies that $f\left(B^{n}\right)$ is a Runge domain; see the remark at the end of page 372 in AL92.

Next, we note the important fact that, given a transition mapping $v_{t}$ and a $G \in S^{0}\left(B^{n}\right)$, then $e^{t} G\left(v_{t}\right)$ is also in $S^{0}\left(B^{n}\right)$, which is mentioned in the proof of Theorem 2.1 in GHKK12.

Lemma 2.6. Let $G \in S^{0}\left(B^{n}\right)$ and $t \geq 0$. Furthermore, let $f \in S^{0}\left(B^{n}\right)$ and $f(z, s)$ be a normalized Loewner chain with $f=f(\cdot, 0)$ such that $\left\{e^{-s} f(\cdot, s)\right\}$ is a normal family. Write $f=f\left(v_{t}, t\right)$. Then $e^{t} G\left(v_{t}\right) \in S^{0}\left(B^{n}\right)$.

Proof. Let $G(z, s)$ be a normalized Loewner chain with $G(\cdot, 0)=G$ such that $\left\{e^{-s} G(\cdot, s)\right\}_{s \geq 0}$ is a normal family and let $F(z, s): B^{n} \times[0, \infty) \rightarrow \mathbb{C}^{n}$ be the mapping

$$
F(z, s)= \begin{cases}e^{t} G(v(z, s, t)), & 0 \leq s \leq t, \\ e^{t} G(z, s-t), & s>t .\end{cases}
$$

Then $F(z, s)$ is a normalized Loewner chain, $F(\cdot, 0)=e^{t} G\left(v_{t}\right)$ and $\left\{e^{-s} F(\cdot, s)\right\}_{s \geq 0}$ is a normal family. Thus $e^{t} G\left(v_{t}\right) \in S^{0}\left(B^{n}\right)$.

Choosing $G(z)=z$ in Lemma 2.6 shows that $e^{t} v_{t} \in S^{0}\left(B^{n}\right)$. Support points of the class $S$ map $B^{1}$ onto $\mathbb{C}$ minus a slit. In particular, they are unbounded mappings. It is not known whether support points of $S^{0}\left(B^{n}\right)$ or $S\left(B^{n}\right)$ can be bounded when $n \geq 2$. Now we will prove Conjecture 2.6 in GHKK12, which says that $e^{t} v_{t}$ is not a support point of $S^{0}\left(B^{n}\right)$ for any $t \geq 0$.

Lemma 2.7. Let $v_{t}$ be defined as in Lemma 2.6 and let $h=e^{t} v_{t} \in S^{0}\left(B^{n}\right)$. Furthermore, let $P \in \mathcal{P}\left(B^{n}, \mathbb{C}^{n}\right)$ with $P(0)=0, D P=0$. Then there exists $\delta>0$ such that $h+\varepsilon e^{t} P\left(e^{-t} h\right) \in S^{0}\left(B^{n}\right)$ for all $\varepsilon \in \mathbb{C}$ with $|\varepsilon|<\delta$.

Proof. Let $g_{\varepsilon}(z)=z+\varepsilon P(z)$. Obviously we have $g_{\varepsilon}(0)=0, D g_{\varepsilon}(0)=I_{n}$. Now $\operatorname{det}\left(D g_{\varepsilon}(z)\right) \rightarrow 1$ for $\varepsilon \rightarrow 0$ uniformly on $\overline{B^{n}}$, so $g_{\varepsilon}$ is locally biholomorphic for $\varepsilon$ small enough. In this case, for every $z \in \overline{B^{n}}$, we have:

$$
\left[D g_{\varepsilon}(z)\right]^{-1}=\left[I_{n}+\varepsilon D P(z)\right]^{-1}=I_{n}-\varepsilon D P(z)+\varepsilon^{2} D P(z)^{2}+\ldots=I_{n}-\varepsilon \underbrace{(D P(z)+\ldots)}_{:=U(z) \in \mathbb{C}^{n \times n}} \text {. }
$$

Write $\left[D g_{\varepsilon}(z)\right]^{-1} g_{\varepsilon}(z)=z+\varepsilon P(z)-\varepsilon U(z) z-\varepsilon^{2} U(z) P(z)=\left(I_{n}+\varepsilon M(z)\right) z$, with a matrix-valued function $M(z)$. Consequently

$$
\left\langle\left[D g_{\varepsilon}(z)\right]^{-1} g_{\varepsilon}(z), z\right\rangle=\left\langle\left(I_{n}+\varepsilon M(z)\right) z, z\right\rangle,
$$

and there is an $\delta>0$ such that $I_{n}+\varepsilon M(z)$ has only eigenvalues with positive real part for all $\varepsilon \in \mathbb{C}$ with $|\varepsilon|<\delta$. In this case we have

$$
\operatorname{Re}\left\langle\left[D g_{\varepsilon}(z)\right]^{-1} g_{\varepsilon}(z), z\right\rangle>0 \quad \forall z \in B^{n} \backslash\{0\},
$$

and thus $g_{\varepsilon} \in S^{*}\left(B^{n}\right) \subset S^{0}\left(B^{n}\right)$ by Theorem 6.2.2 in GK03. From Lemma 2.6 it follows that $e^{t} g_{\varepsilon}\left(v_{t}\right)=e^{t} g_{\varepsilon}\left(e^{-t} h\right)=h+\varepsilon e^{t} P\left(e^{-t} h\right) \in S^{0}\left(B^{n}\right)$.

Proposition 2.8. Let $v_{t}$ be defined as in Lemma 2.6 and let $h=e^{t} v_{t} \in S^{0}\left(B^{n}\right)$. Then $h$ is not a support point of $S^{0}\left(B^{n}\right)$. 
Proof. Let $h=e^{t} v_{t}$ be a support point of $S^{0}\left(B^{n}\right)$; i.e., there is a continuous linear functional $L: \mathcal{H}\left(B^{n}, \mathbb{C}^{n}\right) \rightarrow \mathbb{C}$ such that $\operatorname{Re} L$ is nonconstant on $S^{0}\left(B^{n}\right)$ and

$$
\operatorname{Re} L(h)=\max _{g \in S^{0}\left(B^{n}\right)} \operatorname{Re} L(g)
$$

Let $P$ be a polynomial with $P(0)=0$ and $D P(0)=0$. Then $h+\varepsilon e^{t} P\left(e^{-t} h\right) \in$ $S^{0}\left(B^{n}\right)$ for all $\varepsilon \in \mathbb{C}$ small enough by Lemma 2.7. We conclude $\operatorname{Re} L\left(P\left(e^{-t} h\right)\right)=$ $\operatorname{Re} L\left(P\left(v_{t}\right)\right)=0$; otherwise we could choose $\varepsilon$ such that $\operatorname{Re} L\left(h+\varepsilon e^{t} P\left(e^{-t} h\right)\right)>$ $\operatorname{Re} L(h)$. Now $v_{t}\left(B^{n}\right)$ is a Runge domain by Theorem 2.2 , Hence we can write any analytic function $g$ in the unit ball $B^{n}$ with $g(0)=0$ and $D g(0)=0$ as $g=\lim _{k \rightarrow \infty} P_{k}\left(v_{t}\right)$, where every $P_{k}$ is a polynomial with $P_{k}(0)=0$ and $D P_{k}(0)=0$, according to Lemma $2.1 \mathrm{c}$ ). The continuity of $L$ implies $\operatorname{Re} L(g)=0$. Hence $\operatorname{Re} L$ is constant on $S\left(B^{n}\right)$, a contradiction.

Proof of Theorem 1.1. Let $L$ be a continuous linear functional on $\mathcal{H}\left(B^{n}, \mathbb{C}^{n}\right)$ such that $\operatorname{Re} L$ is nonconstant on $S^{0}\left(B^{n}\right)$ with

$$
\operatorname{Re} L(f)=\max _{g \in S^{0}\left(B^{n}\right)} \operatorname{Re} L(g) .
$$

Fix $t \geq 0$; then $f(z)=f\left(v_{t}(z), t\right)$ for all $z \in B^{n}$. Define the continuous linear functional

$$
J(g):=L\left(e^{t} \cdot g \circ v_{t}\right) \quad \text { for } \quad g \in \mathcal{H}\left(B^{n}, \mathbb{C}^{n}\right) .
$$

Now we have

$$
J\left(e^{-t} f(\cdot, t)\right)=L(f) \quad \text { and } \quad \operatorname{Re} J(g) \leq \operatorname{Re} J\left(e^{-t} f(\cdot, t)\right) \quad \text { for all } \quad g \in \mathcal{H}\left(B^{n}, \mathbb{C}^{n}\right) .
$$

Furthermore, Re $J$ is not constant on $S^{0}\left(B^{n}\right)$ : as $e^{t} v_{t}$ is not a support point of $S^{0}\left(B^{n}\right)$, we have $\operatorname{Re} J(\mathrm{id})=\operatorname{Re} L\left(e^{t} v_{t}\right)<\operatorname{Re} L(f)=\operatorname{Re} J\left(e^{-t} f(\cdot, t)\right)$.

\section{Proof of Theorem 1.2}

In order to simplify notation, we look at only the Roper-Suffridge-Operator $\Psi:=\Psi_{2}$ :

$$
\Psi(f)\left(z_{1}, z_{2}\right)=\left(f\left(z_{1}\right), z_{2} \sqrt{f^{\prime}\left(z_{1}\right)}\right) .
$$

$\Psi$ maps $S$ into $S^{0}\left(B^{2}\right)$. Of course, $\Psi(\operatorname{supp} S) \subseteq \operatorname{supp} \Psi(S)$, because the first coordinate of $\Psi(f)\left(z_{1}, z_{2}\right)$ is just $f\left(z_{1}\right)$.

Now we prove Conjecture 3.1 in GKP07.

Proposition 3.1. Let $f \in S$ be bounded. Then $\Psi(f)$ is not a support point of $\Psi(S)$.

Proof. Let $f \in S$ be bounded and let $L: \mathcal{H}\left(B^{n}, \mathbb{C}^{n}\right) \rightarrow \mathbb{C}$ be a continuous linear functional such that $\operatorname{Re} L$ is nonconstant on $\Psi(S)$ and

$$
\operatorname{Re} L(\Psi(f))=\max _{G \in \Psi(S)} \operatorname{Re} L(G) .
$$


For every $n \geq 1$ there is an $\varepsilon_{0}>0$ such that $f+\frac{\varepsilon}{n+1} f^{n+1} \in S$, for all $\varepsilon \in \mathbb{C}$ with $|\varepsilon|<\varepsilon_{0}$, as $f$ is bounded. It follows that

$$
\begin{array}{r}
\Psi\left(f+\frac{\varepsilon}{n+1} f^{n+1}\right)\left(z_{1}, z_{2}\right)=\left(f\left(z_{1}\right)+\frac{\varepsilon}{n+1} f\left(z_{1}\right)^{n+1}, z_{2} \sqrt{f^{\prime}\left(z_{1}\right)} \sqrt{1+\varepsilon f\left(z_{1}\right)^{n}}\right) \\
=\left(f\left(z_{1}\right)+\frac{\varepsilon}{n+1} f\left(z_{1}\right)^{n+1}, z_{2} \sqrt{f^{\prime}\left(z_{1}\right)}+\varepsilon z_{2} \sqrt{f^{\prime}\left(z_{1}\right)}\left(1 / 2 \cdot f\left(z_{1}\right)^{n}+\ldots\right)\right) \\
=\left(f\left(z_{1}\right), z_{2} \sqrt{f^{\prime}\left(z_{1}\right)}\right)+\varepsilon \underbrace{\left(\frac{1}{n+1} f\left(z_{1}\right)^{n+1}, z_{2} / 2 \sqrt{f^{\prime}\left(z_{1}\right)} f\left(z_{1}\right)^{n}\right)}_{=: P_{n}}+\left(0, \mathcal{O}\left(|\varepsilon|^{2}\right)\right) \in \Psi(S) .
\end{array}
$$

If $\operatorname{Re} L\left(P_{n}\right) \neq 0$, then we can choose $\varepsilon$ such that $\operatorname{Re} L\left(\Psi\left(f+\frac{\varepsilon}{n+1} f^{n+1}\right)\right)>\operatorname{Re} L(\Psi(f))$, a contradiction. Hence,

$$
\operatorname{Re} L\left(\frac{1}{n+1} f\left(z_{1}\right)^{n+1}, z_{2} / 2 \cdot \sqrt{f^{\prime}\left(z_{1}\right)} f\left(z_{1}\right)^{n}\right)=0 \quad \forall n \geq 1 .
$$

Now we can repeat this argument for the $\varepsilon$-terms of higher order, because all coefficients of the expansion $\sqrt{1+x}=1+\frac{1}{2} x \mp \ldots$ are $\neq 0$, and we get

$$
\operatorname{Re} L\left(0, z_{2} \sqrt{f^{\prime}\left(z_{1}\right)} f\left(z_{1}\right)^{j n}\right)=0 \quad \forall j \geq 2, n \geq 1 .
$$

Now consider an arbitrary function of the form $\left(0, z_{2} g\left(z_{1}\right)\right)$ with $g(0)=g^{\prime}(0)=0$. Write $z_{2} g\left(z_{1}\right)=z_{2} \sqrt{f^{\prime}\left(z_{1}\right)} \cdot \frac{g\left(z_{1}\right)}{\sqrt{f^{\prime}\left(z_{1}\right)}}$ and approximate the second factor by a sequence of polynomials in $f$ (see Lemma 2.1 c)):

$$
z_{2} g\left(z_{1}\right)=z_{2} \sqrt{f^{\prime}(z)} \cdot \sum_{k \geq 2} a_{k} f\left(z_{1}\right)^{k}=\sum_{k \geq 2} a_{k} z_{2} \sqrt{f^{\prime}(z)} f\left(z_{1}\right)^{k} .
$$

By using (3.2), it follows that

$$
\operatorname{Re} L\left(0, z_{2} g\left(z_{1}\right)\right)=0 \quad \text { for all } g \in \mathcal{H}\left(B^{1}, \mathbb{C}\right) \text { with } \quad g(0)=g^{\prime}(0)=0 .
$$

We can apply this to (3.1) for $n \geq 2$ to get $\operatorname{Re} L\left(f\left(z_{1}\right)^{n}, 0\right)=0$ for all $n \geq 3$, and by Runge approximation it follows that

(3.4) $\operatorname{Re} L\left(g\left(z_{1}\right), 0\right)=0$ for all $g \in \mathcal{H}\left(B^{1}, \mathbb{C}\right)$ with $g(0)=g^{\prime}(0)=g^{\prime \prime}(0)=0$.

Now let $H\left(z_{1}, z_{2}\right)=\left(h\left(z_{1}\right), z_{2} \sqrt{h^{\prime}\left(z_{1}\right)}\right) \in \Psi(S)$. If $h(z)=z+a_{2} z^{2}+\ldots$, then $\sqrt{h^{\prime}(z)}=\sqrt{1+2 a_{2} z+\ldots}=1+a_{2} z+\ldots$ Thus $\left(h\left(z_{1}\right), z_{2} \sqrt{h^{\prime}\left(z_{1}\right)}\right)=\left(z_{1}+a_{2} z_{1}^{2}+\right.$ $\left.\ldots, z_{2}+a_{2} z_{2} z_{1}+\ldots\right)$ and with (3.3) and (3.4):

$$
\operatorname{Re} L(H)=d_{1}+d_{2} \cdot a_{2}+d_{3}+d_{4} \cdot a_{2}
$$

with $d_{1}=\operatorname{Re} L\left(z_{1}, 0\right), d_{2}=\operatorname{Re} L\left(z_{1}^{2}, 0\right), d_{3}=\operatorname{Re} L\left(0, z_{2}\right), d_{4}=\operatorname{Re} L\left(0, z_{2} z_{1}\right)$. Finally, use (3.1) with $n=1$ to get

$$
\operatorname{Re} L\left(z_{1}^{2}, z_{2} z_{1}\right)=0 \Longleftrightarrow d_{2}+d_{4}=0,
$$

which implies $\operatorname{Re} L(H)=d_{1}+d_{3}$. Hence, $\operatorname{Re} L$ is constant on $\Psi(S)$ and $\Psi(f)$ cannot be a support point of $\Psi(S)$.

Proof of Theorem 1.2. The proof is now quite the same as the proof of Theorem 1.1. We just have to replace $S^{0}\left(B^{n}\right)$ by $\Psi(S)$; see remark 3.1 in GKP07. 


\section{ACKNOWLEDGEMENT}

The author would like to thank the anonymous referee for taking the time to evaluate the manuscript and for providing constructive and valuable comments, in particular for suggesting Corollary 2.4 and Remark 2.5.

\section{REFERENCES}

[ABFW] Leandro Arosio, Filippo Bracci, and Erlend Fornaess Wold, Solving the Loewner PDE in complete hyperbolic starlike domains of $\mathbb{C}^{N}$, eprint arXiv:1207.2721.

[AL92] Erik Andersén and László Lempert, On the group of holomorphic automorphisms of $\mathbf{C}^{n}$, Invent. Math. 110 (1992), no. 2, 371-388, DOI 10.1007/BF01231337. MR.1185588 (93i:32038)

[GHKK12] Ian Graham, Hidetaka Hamada, Gabriela Kohr, and Mirela Kohr, Extreme points, support points and the Loewner variation in several complex variables, Sci. China Math. 55 (2012), no. 7, 1353-1366, DOI 10.1007/s11425-012-4376-0. MR.2943779

[GK03] Ian Graham and Gabriela Kohr, Geometric function theory in one and higher dimensions, Monographs and Textbooks in Pure and Applied Mathematics, vol. 255, Marcel Dekker Inc., New York, 2003. MR2017933(2004i:32002)

[GKP07] Ian Graham, Gabriela Kohr, and John A. Pfaltzgraff, Parametric representation and linear functionals associated with extension operators for biholomorphic mappings, Rev. Roumaine Math. Pures Appl. 52 (2007), no. 1, 47-68. MR2341607(2008i:32018)

[HM84] D. J. Hallenbeck and T. H. MacGregor, Linear problems and convexity techniques in geometric function theory, Monographs and Studies in Mathematics, vol. 22, Pitman (Advanced Publishing Program), Boston, MA, 1984. MR768747 (86c:30016)

[Koh01] Gabriela Kohr, Using the method of Löwner chains to introduce some subclasses of biholomorphic mappings in $\mathbf{C}^{n}$, Rev. Roumaine Math. Pures Appl. 46 (2001), no. 6, 743-760 (2002). MR1929522 (2003h:32022)

[Wer59] John Wermer, An example concerning polynomial convexity, Math. Ann. 139 (1959), 147-150 (1959). MR0121500 (22 \#12238)

Department of Mathematics, University of Wuerzburg, 97074 Wuerzburg, Germany

E-mail address: sebastian.schleissinger@mathematik.uni-wuerzburg.de 